\section{AB0570 MIXED CONNECTIVE TISSUE DISEASE: NOT THAT UNCOMMON, A SINGLE-CENTER EXPERIENCE FROM INDIA}

V. Dhir ${ }^{1}$, S. Jha ${ }^{1}$, A. Sharma ${ }^{1}$, S. Jain ${ }^{1}$, S. K. Sharma ${ }^{1}$, G. Naidu ${ }^{1} .{ }^{1}$ Post Graduate Institute of Medical Education \& Research, Chandigarh, Chandigarh, India

Background: Mixed connective tissue disease (MCTD) is considered to be uncommon; specifically there is sparse data on MCTD from developing countries like India.

Objectives: This study examines the clinical and serological features of these patients in a single center in North-India.

Methods: This was a retrospective single-center study of patients diagnosed as MCTD in last 20 years. The patients included fulfilled at least one of the diagnostic criteria namely Alarcón-Segovia, Kasukawa, and Kahns. Demographic details, clinical signs and symptoms, laboratory parameters, treatment and outcome were extracted from medical records and clinic files in a pre-designed proforma.

Results: This study included 41 MCTD patients. There was a marked female preponderance $(\mathrm{F}: \mathrm{M}=40: 1)$, and mean age of disease onset and diagnosis was $33.8 \pm 10.7$ and $39.3 \pm 10.2$ years. $39(92 \%)$ of the patients fulfilled both Kahn and Kasukawa criteria, while $31(76 \%)$ fulfilled Alarcon-Segovia criteria. Initially patients had been (mis)diagnosed as rheumatoid arthritis, systemic lupus erythematosus (or UCTD) (in five patients each), overlap syndromes or myositis (in 4 patients). ANA was commonly high-titer and specked, U1RNP was positive in all. (Table 1) Other autoantibodies on immunoblot included SSA and Ro52 in half the patients. Raynaud's was seen in three-fourth at presentation and all the patients over time. Digital gangrene and puffy fingers were seen in $8(20 \%)$ and $18(46 \%)$ patients. Other clinical features included arthritis in $33(81 \%)$, sclerodactyly in $23(56 \%)$ and proximal weakness in 20 patients $(49 \%)$. Interstitial lung disease and pulmonary arterial hypertension were seen in $20(57 \%)$ and $15(44 \%)$ patients. All patients (except one) received prednisolone, and it was currently used in almost $90 \%$. Intravenous cyclophosphamide was used in onethird, commonly for ILD.

Table 1. Laboratory features of patients with MCTD

\begin{tabular}{|c|c|}
\hline Labs n (\%) & \\
\hline Leucopenia & $9(22)$ \\
\hline Thrombocytopenia & $10(24)$ \\
\hline Raised globulins $^{\mathrm{b}}$ & $22(69)$ \\
\hline Mean globulins, mean (SD) ${ }^{\mathrm{a}}$ & $5 \pm 3.4$ \\
\hline Elevated CPK ${ }^{\mathrm{b}}$ & $10(31)$ \\
\hline CPK Levels U/L, median (IQR) & $256(57.5-1036)$ \\
\hline ANA Speckled Pattern N (\%) & $31^{d}(82)$ \\
\hline U1RNP N (\%) & $32(100 \%)$ \\
\hline \multicolumn{2}{|l|}{ U1RNP Blot Intensity } \\
\hline $1+$ & $8(25 \%)$ \\
\hline $2+$ & $1(3.2 \%)$ \\
\hline $3+$ & $7(21.8 \%)$ \\
\hline $4+$ & $16(50 \%)$ \\
\hline U1RNP EIA, mean(SD), n=19 & $141.3 \pm 82.4$ \\
\hline Raised RF Titers ${ }^{h}$ & $10(35.7 \%)$ \\
\hline Low C3 mg/dlf & $8(40 \%)$ \\
\hline Low $\mathrm{C} 4 \mathrm{mg} / \mathrm{dl}^{f}$ & $3(15 \%)$ \\
\hline FVC, mean (SD) $n=20$ & $82.4 \pm 18.9$ \\
\hline ILD on $\mathrm{HRCT}^{9}$ & $20(57)$ \\
\hline Dilated PA on $\mathrm{CT}^{f}$ & $10(50)$ \\
\hline $\mathrm{PAH}^{e}$ on $\mathrm{ECHO}$ & $15(44.1 \%)$ \\
\hline RA/RV Dilated & $5(18)$ \\
\hline
\end{tabular}

SD-standard deviation, IQR- Interquartile range, CPK- Creatinine phosphokinase,, ILDInterstitial lung disease, PAH- Pulmonary arterial hypertension, ANA- Anti nuclear antibody, IIF- Indirect Immunofluorescence, PA- pulmonary artery, RA/RV- Right atrium/Right Ventricle, ${ }^{\mathrm{a}} 34^{\mathrm{b}} 32^{\mathrm{d}} 38^{\mathrm{e}} 34^{\mathrm{f}} 20^{\mathrm{g}} 34^{\mathrm{h}} 28$

Conclusion: MCTD was not uncommon in the single-center in North India. Kahn and Kasukawa criteria were found to be the most sensitive for its diagnosis. Digital gangrene was relatively common and sometimes the presenting feature; whereas puffy fingers was present in only half the patients.

Disclosure of Interests: None declared

DOI: 10.1136/annrheumdis-2020-eular.5530

\section{$\mathrm{AB} 0571$ \\ IS RITUXIMAB AN ADEQUATE GLUCOCORTICOID SPARING AGENT IN IDIOPATHIC INFLAMMATORY MYOPATHIES?}

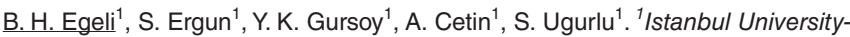
Cerrahpasa, Istanbul, Turkey
Background: Idiopathic inflammatory myopathies (IIM) are essentially treated aiming improvement of muscle function and extra muscular disease manifestations. The backbone of the treatment is corticosteroids enhancing the survival and patient quality of life. The lack of consensus on target-specific immunosuppressive treatment highlights the need for further studies evaluating alternative treatment methods. Rituximab is potentially a glucocorticoid-sparing agent which was reviewed in multiple studies with small sample sizes due to the rarity of the disease.

Objectives: Higher statistical power can enhance the trustworthiness of alternative treatment methods yielding the main objective of this study.

Methods: This retrospective study was conducted at a tertiary rheumatology center. Patients were diagnosed with an idiopathic inflammatory myopathy (dermatomyositis [DM], polymyositis [PM]) and were treated with rituximab in order to be included in this study. Clinical signs and symptoms of the presentation were noted during the first patient encounter as well as the follow-up. Parameters of disease activity including acute phase reactants, muscle enzyme levels, and disease-specific autoantibodies were analyzed.

Results: The study includes 28 patients (20 DM, $8 \mathrm{PM}$ ). The age of diagnosis was $43.44 \pm 15.77$ years, follow-up duration was $60.7 \pm 70.7$ months. The presenting signs and symptoms of the patients are shown in Figure 1. The parameters of disease activity before and after treatment are summarized in Table 1. The mean corticosteroid dose decreased from $31.429 \pm 23.934 \mathrm{mg}$ to $10.278 \pm 12.001(p=0.001)$. Other treatment methods were methotrexate $(\mathrm{n}=18)$, Intravenous Immunoglobulin (IVIG) $(\mathrm{n}=7)$, and cyclophosphamide $(n=2)$. There were not any deaths during the follow-up. Two patients were lost to follow-up.

Table 1. The Parameters of Disease Activity Before and After Treatment

\begin{tabular}{lccc}
\hline & Before Treatment & After Treatment & P Value \\
\hline CPK, mean \pm std (U/L) & $1426 \pm 2049.92$ & $263.44 \pm 265.63$ & 0.004 \\
LDH, mean \pm std (U/L) & $557.5 \pm 365$ & $379.78 \pm 192.1$ & 0.03 \\
AST, mean \pm std (U/L) & $62.52 \pm 59$ & $30.16 \pm 27.59$ & 0.01 \\
ALT, mean \pm std (U/L) & $56.48 \pm 49.21$ & $27.64 \pm 24.52$ & 0.008 \\
ESR, mean \pm std (mm/hour) & $26.38 \pm 28.98$ & $20.39 \pm 18.76$ & 0.36 \\
CRP, mean \pm std (mg/L) & $19.23 \pm 46.15$ & $12.53 \pm 26.67$ & 0.5 \\
RF, mean \pm std (U/mL) & $0(0)$ & $\mathrm{N} / \mathrm{A}$ & $\mathrm{N} / \mathrm{A}$ \\
ANA, $\mathbf{n}(\%)$ & $3(10.71)$ & $\mathrm{N} / \mathrm{A}$ & $\mathrm{N} / \mathrm{A}$ \\
\hline
\end{tabular}

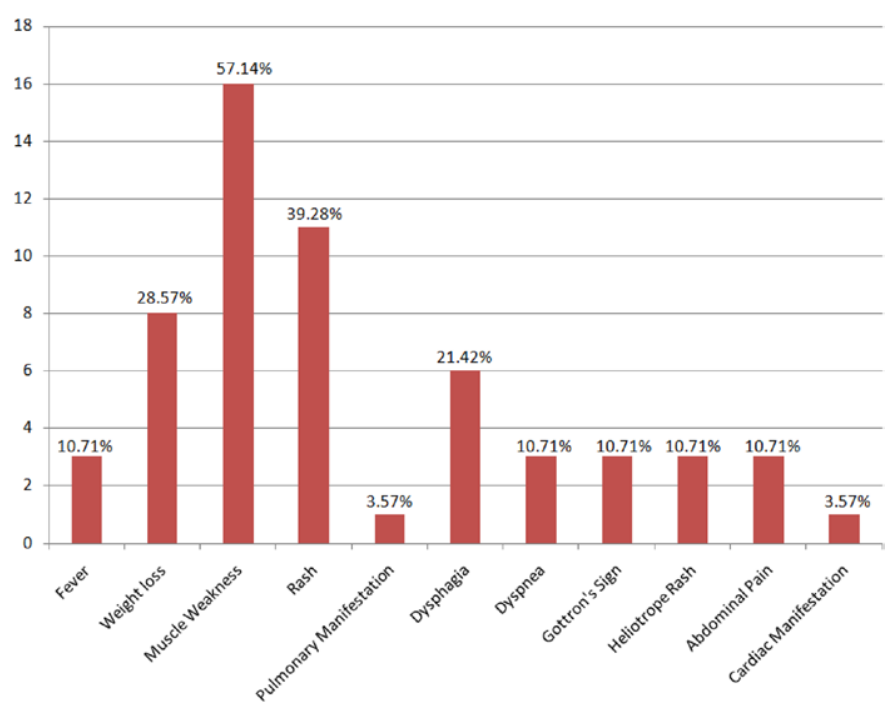

Figure 1. The Presenting Signs and Symptoms of the Patients

Conclusion: Rituximab is shown to be effective in treating myositis along with corticosteroids as well as a corticosteroid-sparing agent in retrospective studies and open-label clinical trials; however, lack of statistical power should be underlined. Long term decrease in steroid use and decrease in disease activity markers hints the effective use of rituximab as a glucocorticoid sparing agent as well as its safety with minimal side effects.

Disclosure of Interests: None declared

DOI: 10.1136/annrheumdis-2020-eular.4038 